\title{
Can models based on Artificial neural networks help predict the risk of fracture in osteoporotic patients?
}

\author{
Authors: Matanović Luka', Mišura Damir², Štajduhar Andrija (mentor) \\ ${ }^{1}$ University of Zagreb, School of Medicine, Zagreb, Croatia \\ ${ }^{2}$ Department of Medical Statistics of University of Zagreb, School of Medicine, Zagreb, Croatia \\ DOI: https://doi.org/10.26800/LV-142-supp5-60
}

\section{Introduction:}

Osteoporosis is a systemic skeletal disorder characterized by low bone mass, leading to bone fragility, and consequent increase in fracture risk. Globally osteoporosis affects more than 200 million people, being the cause of more than 8.9 million fractures annually. Currently the most used diagnostic tool to predict 10 -year probability of bone fracture risk is FRAX.

\section{Aim:}

In this article we investigate the literature on whether the use of models based on Artificial neural network (ANN) can provide better estimates of the risk of fracture.

\section{Materials And Methods:}

We reviewed full text articles published on PubMed in the last 5 years $(2015$ - 2020).

\section{Results:}

The usage of ANNs in estimating risk of fracture in osteoporotic patients is still debatable. ANNs can be used in predicting risk using standard tools such as FRAX or OSTA where they show slight advantage over linear regression. Another possibility is to use ANNs in processing radiological images of various modalities. To demystify the potential of this approach more studies are required and the success of these models depend on the learning dataset (the bigger the sample, the better the model will be).

\section{Conclusion:}

ANNs can be very helpful when searching for subtle patterns in the radiological images that characterize bone degradation processes are important to recognize early in order to establish a reliable risk estimate. All the publications covered in this article would benefit from bigger samples, including more participants in the study, especially male participants. Furthermore, samples should be more heterogeneous, including subjects of different ethnicity, race etc. We would also like to highlight the importance of data transparency and objectivity in researches. Since neural networks function similar to blackboxes, the obtained outputs are often difficult to interpret by humans. Therefore, it is important to develop methods that provide objective quantification and comparison between different models based on ANNs.

Keywords: artificial neural network, fracture risk, osteoporosis, systematic review 